Final version at Synthese, DOI: 10.1007/s11229-015-0754-9

\title{
CONDITIONAL CHOICE WITH A VACUOUS SECOND TIER
}

\author{
RUSH T. STEWART \\ DEPARTMENT OF PHILOSOPHY, COLUMBIA UNIVERSITY
}

\begin{abstract}
This paper studies a generalization of rational choice theory. I briefly review the motivations that Helzner gives for his conditional choice construction (Helzner, 2013). Then, I focus on the important class of conditional choice functions with vacuous second tiers. This class is interesting for both formal and philosophical reasons. I argue that this class makes explicit one of conditional choice's normative motivations in terms of an account of neutrality advocated within a certain tradition in decision theory. The observations recorded - several of which are generalizations of central results in the standard theory of rational choice - are intended to provide further insight into how conditional choice generalizes the standard account and are offered as additional evidence of the fruitfulness of the conditional choice framework. Rational Choice and Decision Theory and Uncertainty and Value Conflict and Conditional Judgment
\end{abstract}

\section{Admissibility and Preference}

For the received view of rationality, the concept of preference is central. This is true, too, for the expectation tradition in decision theory more generally, from early accounts of expected value to Savage's widely endorsed development of subjective expected utility theory (Savage, 1972, originally published in 1954; Helzner, MS). ${ }^{1}$ According to the subjective expected utility tradition's account of decision making, a rational agent has a credal state that can be represented by a probability distribution over the relevant state space and values that can be represented by a cardinal utility function over the relevant space of outcomes. What rationality demands is that an agent's choices maximize expectation with respect to the indicated sort of probability and utility functions (Savage, 1972, originally published in 1954; Luce and Raiffa, 1957). Subjective expected utility induces a preference ordering on the set of alternatives and rational choices are those made in accordance with that ordering (or, provided an agent's preferences satisfy certain constraints, she can be represented as maximizing expected utility). Even many deviations from this view - from descriptive theories such as Kahneman and Tversky's prospect theory (1979) to normative accounts such as those of Ellsberg (1963) and Gärdenfors and Sahlin (1982) - retain the assumption of a preference ordering while surrendering other aspects of the expected utility tradition.

But there is also a "persistent underground movement" that calls into question the normative status of the ordering assumption for preference (Seidenfeld, 1988).

Date: Received: 14 October 2014 / Accepted: 15 April 2015.

Thanks are due to John Collins, Jeff Helzner, Tobias Lessmeister, Isaac Levi, Yang Liu, Ignacio Ojea, Paul Pedersen, Hans Rott, and two anonymous referees for helpful comments and discussions.

${ }^{1}$ As Helzner points out, while Savage's work eximplifies the tradition of expected utility most familiar to economists, psychologists, and statisticians, perhaps the tradition descending from Richard Jeffrey (1983) is most well-known among philosophers. 
These theorists are motivated by, among other things, many of the same concerns that motivate the deviations mentioned in the previous paragraph, but point the finger at ordering as the problem. The fact that sophisticated decision-makers under little cognitive load endorse, even upon reflection, choices that do not satisfy the demands of subjective expected utility theory in cases like those Ellsberg presents (1963), for example, is taken by many to be grounds to explore descriptive and normative alternatives.

Perhaps most prominent of such concerns has to do with the received view's treatment of uncertainty. In Games and Decisions, Luce and Raiffa treat decisions according to the now-famous tripartite distinction: decisions under certainty, decisions under risk, and decisions under uncertainty (1957, p. 13). In decisions under certainty, "each action is known to lead invariably to a specific outcome." In decisions under risk, the agent has access to an objective probability distribution over the relevant state space. By contrast, decision-making under uncertainty obtains when the agent does not have such access, either because the probabilities "are completely unknown or are not even meaningful." According to the subjective expected utility tradition, the distinction between risk and uncertainty is not particularly meaningful because, even if statistical information is unavailable, the agent still has access to a determinate probability distribution over the state space; the probabilities just are not objective. Many view the reduction of uncertainty to a single subjective probability distribution as problematic, including Keynes in his discussion of the weight of evidence (1921), Knight with his notion of unmeasurable uncertainty (1921), Ellsberg in his critique of the Savage axioms (1963), Kyburg in his criticism of the subjective interpretation of probability (1968), and Levi with his normative conception of doubt (1974). More recent contributions to alternative accounts of uncertainty in decision theory include those of Walley (1991), Kadane et al (1999), and Seidenfeld et al (2010).

Consider the urn of Ellsberg's three color problem. What is known about the composition of the urn is that it contains exactly 90 balls, 30 of which are red, the remainder an unknown mixture of yellow and blue balls. There are 61 possible mixture compositions of the urn consistent with this information. A ball is to be drawn at random. What probabilities are to be assigned to the three possible outcomes? The standard Bayesian account rules out numerous states of suspense in probabilistic judgment among all of the statistical hypotheses consistent with the given information. The rational agent is assumed to have a credal state representable by a single probability distribution, perhaps by some particular statistical distribution, or by an assignment of sharp credal probabilities to the statistical hypotheses. For a bet on the outcome of the draw, the rational agent, on this account, is obliged to maximize expected utility with respect to that single probability distribution. Regarding two or more distributions as permissible to use in inference and decision making is a foreclosed possibility.

In contrast, Isaac Levi denies that in such cases agents are obliged to be in credal states representable by a single probability function. We might instead take the set of probability functions corresponding to the statistical hypotheses compatible with the evidence about the urn to represent a legitimate credal state. Levi correspondingly advocates a decision rule for sets of probability functions 
(Levi, though, requires convexity of states of subjective probability). ${ }^{2}$ For a set $\mathbf{P}$ of probability functions and a set $\mathbf{U}$ of utility functions, the $E$-admissible options are those that maximize expected utility for some $(p, u) \in \mathbf{P} \times \mathbf{U}$. Every probability function in $\mathbf{P}$ is permissible to use in assessing expected utilities. It is easy to see that $E$-admissibility generates a set of orderings of the options that will, in general, disagree in some respects. Such a decision theory does not induce a preference ordering over the options.

Instead of recommending the options that are optimal according to a (single) preference ordering, E-admissibility generalizes to a notion of admissibility, or acceptability for choice. In some cases, admissibility and preference coincide, but admissibility is the more general notion. Admissible options are those that are consistent with the agent's relevant commitments, those options that the agent's commitments fail to rule out for choice (Nehring, 1997; Helzner, 2011, MS; Rubinstein, 2012). For decision making, the relevant sorts of commitments include values and beliefs. A (non-singleton) set of probability functions represents a weaker state of credal probability judgment than does a single probability function, so, in general, more options will be consistent with the weaker state, as the $E$-admissibility rule attests.

Other sources of indeterminacy, such as conflict in values (indeterminacy in utility), lead to analogous problems with ordering, and, as Sen argues, there are other reasons to think that the relationship between preference and choice is often subtle and complex (1997). It seems reasonable, then, to seek a more general, unifying, and neutral setting in which to investigate many aspects of rational choice, including, in particular, departures from ordering (Helzner, MS). The framework of set-valued choice functions provides such a setting. After briefly reviewing the standard account of rational choice, I turn to conditional choice (Helzner, 2013). Conditional choice generalizes the standard account, moving to conditional judgments of admissibility. Among the motivations I recount in Section 3 is that a natural characterization of two-tiered choice is available in the conditional choice setting, where one is lacking in the standard framework of choice functions. Rules like $E$-admissibility recommend generally permissive judgments of acceptability for choice. Secondary (tertiary, etc.) criteria can be invoked to contract the firsttier admissible options further. Even in cases in which either there is no relevant second-tier criterion or the options are tied with respect to such a criterion (that is, cases in which the second-tier is vacuous), the conditional choice framework is still both formally and philosophically interesting. This special case inherits many of the philosophical motivations for conditional choice in general. As I argue in Section 4, a normative account of neutrality or suspense among candidate ways of evaluating options that underwrites conditional choice can be clearly expressed. Furthermore, many of the interesting connections between rational choice and belief revision theory observed by Hans Rott are preserved when the second tier is vacuous (remarks in Section 4). Finally, in addition to several other connections to the standard theory of rational choice (Observations 2, 6, and 8), generalizations and alternative characterizations of the notion of pseudo-rationalizability are available (Observations 9, 10, and 11).

\footnotetext{
${ }^{2}$ Seidenfeld et al.'s account of coherent choice generalizes Levi's account by relaxing convexity (2010).
} 


\section{Choice Functions}

Let $X$ be a set of alternatives or options. Let $\mathcal{X}$ be a collection of subsets of $X$. Intuitively, $\mathcal{X}$ is the set of menus from which an agent is to choose, carved from the universal set of alternatives, $X$. Sometimes additional assumptions are made about $\mathcal{X}$. For example, we could stipulate that $\mathcal{X}$ be closed under finite or arbitrary unions or that $\mathcal{X}$ be compact (for every element of $\mathcal{X}$, if that element is a subset of the union of some collection of elements of $\mathcal{X}$, then that element is a subset of the union of some finite subset of the collection). For the purposes of this paper, we will generally assume that $\mathcal{X}$ consists of all the finite subsets of $X$. The concern with this distinguished domain for choice is customary in the literature (Sen, 1971; Suzumura, 1983; Moulin, 1985; Rott, 2001; Helzner, 2013).

Definition 1. A choice function is a function $C: \mathcal{X} \rightarrow \mathscr{P}(X)$ such that $C(Y) \subseteq Y$ for every $Y \in \mathcal{X}{ }^{3}$

$C(Y)$ is called the choice set. In this paper, it is not assumed, as it typically is, that choice sets are non-empty. More will be said about relaxing this assumption later on.

Instead of preference, the central concept is the more general notion of admissibility. A choice function partitions a set of options into the set of admissible options and the complementary subset of rejected or inadmissible options. So, given a menu, $Y$, a choice function returns the set of admissible options, $C(Y)$. Cases in which judgments of admissibility are reducible to a preference ordering can be characterized in terms of rationality constraints on choice functions. We turn to such constraints now.

2.1. Choice Constraints and Rationalizable Choice. So that's what a choice function is. But which choice functions are the rational ones? In the classical theory of choice, there is a slogan that answers that question: "Rational choice is relational choice" (Rott, 2001, p. 154). The idea is that a choice function is rational just in case it can be regarded as based on some underlying binary relation.

Definition 2. A binary relation $R$ on $X$ rationalizes $C$ if, for every $Y \in \mathcal{X}$,

$$
C(Y)=\{x \in Y: x R y \text { for all } y \in Y\} .
$$

And $C$ is said to be rationalizable. For a rationalizable choice function, admissibility in a menu is determined by pairwise comparisons (under the relation on $X$ ) of the menu's elements.

There are two kinds of relations that are standardly considered in this context. One is a non-strict (reflexive) relation. The other is strict (asymmetric).

(O) $C(Y)=\{x \in Y: x \geqslant y$ for all $y \in Y\}$

(M) $C(Y)=\{x \in Y: y>x$ for no $y \in Y\}$

The first formalization is called optimization, the second, maximization (Sen, 1997). Maximization is the more general notion, applying even in cases in which $R$ is not complete (that is, even in scenarios in which it is not the case that any two alternatives can be compared). ${ }^{4}$

\footnotetext{
$3 \mathscr{P}(X)$ is the power set of $X$.

${ }^{4}$ Though we can define a relation $\geqslant$ by $x \geqslant y$ iff $\neg(y>x)$ that rationalizes the choice function in the precise sense specified above. Note that this relation generated from $>$ is complete, converting incompleteness into indifference.
} 
The following is a list of coherence constraints on choice functions. The conditions are abstract and intended to apply to any choice function (whether it is determined by a probability/utility pair or something very different).

(I) If $Y \subseteq Y^{\prime}$, then $Y \cap C\left(Y^{\prime}\right) \subseteq C(Y)$

$\left(I^{-}\right) \quad$ If $Y \subseteq Y^{\prime}$ and $C\left(Y^{\prime}\right) \subseteq Y$, then $C\left(Y^{\prime}\right) \subseteq C(Y)$

$\left(I^{\prime}\right) \quad C\left(Y \cup Y^{\prime}\right) \subseteq C(Y) \cup C\left(Y^{\prime}\right)$

(II) $C(Y) \cap C\left(Y^{\prime}\right) \subseteq C\left(Y \cup Y^{\prime}\right)$

$\left(I I^{+}\right) \quad$ If $x \in C(Y)$ and $y \in C\left(Y^{\prime}\right)$, then $x \in C\left(Y \cup Y^{\prime}\right)$ or $y \in C\left(Y \cup Y^{\prime}\right)$

(III) If $Y \subseteq Y^{\prime}$ and $C\left(Y^{\prime}\right) \subseteq Y$, then $C(Y) \subseteq C\left(Y^{\prime}\right)$

$(I V)$ If $Y \subseteq Y^{\prime}$ and $C\left(Y^{\prime}\right) \cap Y \neq \varnothing$, then $C(Y) \subseteq C\left(Y^{\prime}\right)$

$\left(I V^{+}\right) \quad$ If $Y \subseteq Y^{\prime}$, then $C(Y) \subseteq C\left(Y^{\prime}\right)$

(Faith1) $\forall Y \in \mathcal{X}:$ if $Y \cap B \neq \varnothing$, then $C(Y) \subseteq B$

(Faith2) $\forall Y \in \mathcal{X}: Y \cap B \subseteq C(Y)$

(Success) If $Y \neq \varnothing$, then $C(Y) \neq \varnothing$

$(\varnothing 1) \quad$ If $Y \subseteq Y^{\prime}$ and $C\left(Y^{\prime}\right)=\varnothing$, then $C(Y)=\varnothing$

$(\varnothing 2) \quad$ If $Y \subseteq Y^{\prime}$ and $C(Y)=\varnothing$, then $C\left(Y^{\prime}\right) \cap Y=\varnothing$

Many of these constraints are standard. ${ }^{5}$ Some Hans Rott suggests in his Change, Choice and Inference (2001) and are included here because of their relevance to future work connecting rational choice to belief revision. The naming system adopted here is Rott's. $(I)$ is Sen's Property $\alpha,(I I)$ a finitary version of Property $\gamma$. (III) is Aizerman's Axiom and is of more recent pedigree than Sen's properties. $(I V)$ is Sen's Property $\beta^{+}$.

There is an important aspect of such constraints that deserves comment. Every constraint (except (Success) - more on that below) mentions more than one menu: they are intermenu constraints. But such standards are intended to be synchronic. This has to do with the hypothetical nature of choice functions. The idea is that judging certain options to be admissible on one menu rationally commits one to hypothetically judging a certain way on another menu at that time (that is, with no changes in admissibility-determining judgments). Consider $(I)$, alternatively called Sen's Property $\alpha$, Chernoff, and Heritage. According to Property $\alpha$, a rational choice function is such that, if an option is judged admissible on a menu, then that option remains admissible on any subset of the menu that includes it. Merely removing options, in other words, ought not to demote an alternative from being judged admissible - so long as the parameters generating the choice function are held fixed.

Importantly, given particular domain restrictions, that is, restrictions on $\mathcal{X}$, satisfying a certain small set of choice constraints guarantees rationalizability for a choice function. The following theorem is a central result in this area. Instead of the more standard presentation (see (Sen, 1971)), I will give Rott's slightly more complicated version of the theorem.

Theorem 1. Let $C$ be a choice function satisfying $(\varnothing 1)$, where $\mathcal{X}$ is the set of all finite subsets of $X . C$ is rationalizable iff it satisfies $(I)$ and $(I I){ }^{6}$

\footnotetext{
${ }^{5}$ In the non-standard Faith constraints, $B$ is intended to represent a set of "absolutely satisfactory options." Together, the Faith constraints entail that all and only absolutely satisfactory options are in the choice set when present on a menu.

${ }^{6}$ See (Rott, 2001, pp.286-290) for proof.
} 
The reason for the more complicated statement of the result is that Rott is concerned about domain conditions and refusals to choose. For one thing, in Rott's version, $\mathcal{X}$ contains the empty set. For another, our definition of a choice functionwhich is also Rott's - does not imply that choice functions automatically satisfy (Success). If, pace Rott, we insist on (Success) and ensure $\varnothing \notin \mathcal{X}$, then Theorem 1 holds for the domain of all non-empty, finite subsets of $X$ just by imposing $(I)$ and $(I I)$ (the more standard formulation); stipulating that $C$ satisfies $(\varnothing 1)$ is unnecessary. This is easy to see because $(\varnothing 1)$ and $(\varnothing 2)$ are vacuously satisfied since it is never the case that $C(Y)=\varnothing$.

Cases in which judgments of admissibility are reducible to a preference ordering can also be characterized by conditions on choice functions. When $C$ satisfies Sen's well known-properties $\alpha$ and $\beta^{+}$, or $(I)$ and $(I V), C(Y)$ can be interpreted as the elements that are optimal in $Y$ with respect to the underlying preference relation (1971)..$^{7}$

Theorem 2. Let $C$ be a choice function satisfying $(\varnothing 1)$, where $\mathcal{X}$ is the set of all finite subsets of $X . C$ is weak order rationalizable iff it satisfies $(I)$ and $(I V)$.

The standard proofs of the central results presented in this section make use of the notion of revealed preference. The idea is to recover an underlying preference relation from the choice function. There are a number of ways to define a revealed preference relation, $R_{C} \subseteq X \times X$. Though in general such relations are not equivalent, given certain typical assumptions-e.g., that the domain consists of all and only the finite subsets of $X$ and that the choice functions satisfy certain constraints - the following two are equivalent in the relevant contexts. The first is called Samuelson preference

$$
x R_{C^{s}} y \text { iff, for some } Y \in \mathcal{X}, x \in C(Y) \text { and } y \in Y
$$

and the second is called base preference

$$
x R_{C^{b}} y \text { iff } x \in C(\{x, y\}) .
$$

Sen calls a choice function normal if its revealed preference relation, when used as the basis for choice, regenerates the choice function itself (1971). Normality is equivalent to rationalizability. For non-binary choice functions, the two relations do not coincide.

2.2. Pseudo-Rationalizability. Relaxing ordering is one level of heresy; there are reasons, however, to consider relaxing even binariness of choice (Sen, 1993; Nehring, 1997). In fact, the decision theory advocated by Levi (1974), for example, and its generalization in the hands of Seidenfeld, et al. (2010), do not reduce to binary comparisons between options.

Consider the following set of alternatives for Ellsberg's urn:

The outcomes are dollar amounts. Assume that the agent's utilities are determinate and linear in dollars. Not imposing convexity, take the agent's credal state to correspond to the set of configurations of the urn consistent with the evidence. So, $\mathbf{P}$ is the set of probability functions on $\{$ Red, Yellow, Blue $\}$ such that $p($ Red $)=$ $\frac{1}{3}, p($ Yellow $)=\frac{n}{90}, p$ (Blue $)=\frac{60-n}{90}$ for some natural number $n \leqslant 60$. Consider $(I V) .\{f, h\} \subseteq\{f, g, h\}$ and $f$ is $E$-admissible in $\{f, g, h\}$ and $\{f, h\}$, so $C(\{f, g, h\}) \cap$

\footnotetext{
${ }^{7}$ In (Sen, 1971), Sen uses $\beta$ instead of the stronger condition $\beta^{+}$. In the presense of $\alpha$, however, $\beta$ and $\beta^{+}$are equivalent. $\beta$ : If $x, y \in C(Y), Y \subseteq Y^{\prime}$, and $y \in C\left(Y^{\prime}\right)$, then $x \in C\left(Y^{\prime}\right)$.
} 
TABLE 1. Options for Ellsberg's Urn (Helzner, 2013)

\begin{tabular}{llll}
\hline & Red & Yellow & Blue \\
\hline$f$ & 3 & 0 & 3 \\
$g$ & 3 & 3 & 0 \\
$h$ & $3 / 2$ & $3 / 2$ & $3 / 2$ \\
\hline
\end{tabular}

$\{f, h\} \neq \varnothing$. But $h$ is $E$-admissible in $\{f, h\}$ while it is not in $\{f, g, h\}$, so $C(\{f, h\}) \nsubseteq$ $C(\{f, g, h\})$. $(I V)$ is violated, so judgments of admissibility do not reduce to an ordering of the options, as indicated above.

Now consider $(I I)$. We have seen that $h$ is $E$-admissible in $\{f, h\}$, but it is also $E$-admissible in $\{g, h\}$. However, we have also seen that $h$ is not $E$-admissible in $\{f, g, h\} . C(\{f, h\}) \cap C(\{g, h\}) \nsubseteq C(\{f, g\} \cup\{g, h\})$. (II) is violated, so judgments of admissibility do not reduce to binary comparisons of the options either.

Within the literature on choice functions, weaker notions than rationalizability by a binary relation have been considered. One such notion is called collected extremal choice by Aizerman and Malishevski (1981) and pseudo-rationalizability by Moulin (1985).

Definition 3. A choice function $C$ is pseudo-rationalizable if there is a finite collection $\left\{C_{i}: i \in I\right\}$ of weak order rationalizable choice functions such that, for all $Y \in \mathcal{X}$

$$
C(Y)=\bigcup_{i \in I} C_{i}(Y)
$$

For every menu, $C$ returns the options that are best according to each $C_{i}$. Loosely speaking, $E$-admissibility and Seidenfeld et al.'s "coherent choice" rule both determine pseudo-rationalizable choice functions. Each probability/utility pair in $\mathbf{P} \times \mathbf{U}$ determines a weak order rationalizable choice function. Since an option is admissible just in case it has a witnessing probability/utility pair, admissibility according to these rules can be construed as the union of a (not necessarily finite) collection of weak order rationalizable choice functions. As Aizerman and Malishevski show, pseudo-rationalizable choice functions can be characterized in terms of simple coherence constraints.

Theorem 3. Let the set $X$ of options be finite and let $\mathcal{X}$ be the set of all non-empty subsets of $X$. A choice function $C$ on $\mathcal{X}$ is pseudo-rationalizable iff it satisfies (I) and $(I I I)$.

As an alternative rationalization concept, pseudo-rationalizability, as we will see (Observations 2, 9, and 11), is very closely related to the choice structure of concern in what follows. The account that emerges will be closely related to pseudo-rationalizable choice functions, but in some ways more general, and in other ways more structured. The general account of conditional choice is introduced and motivated over the course of the next two sections. Then, attention is turned to the special case of conditional choice with a vacuous second tier and connections to rationalizability and pseudo-rationalizability.

\section{Conditional Choice}

Jeff Helzner has recently proposed conditional choice as a generalization of the classical framework of rational choice (2013). Roughly speaking, conditional choice 
encodes conditional judgments of admissibility. The judgments are conditional on antecedent, admissibility-determining judgments, such as beliefs or values (or both). To take a particular interpretation of the conditioning set, an agent might deem one set of options as admissible relative to a set $\mathbf{P}$ of probability functions and another set admissible relative to $\mathbf{P}^{\prime} \neq \mathbf{P}$. An exact definition is provided below.

This section collects some of the motivations that have been given in various places for this construction. One motivation for the move to conditional choice is the one Helzner is concerned with in that first paper, namely, rationalizing two-tiered choice. Decision rules that first apply one criterion to the menu and then a second criterion to the set of options compatible with the first criterion, whittling the admissible options down further, define a two-tiered choice function. For example, after restricting choice to the $E$-admissible options on the menu, an agent could then apply a second-tier security criterion. The resulting set of admissible options would be those $E$-admissible options that also have the best worst-case outcomes evaluated with respect to $\mathbf{P} \times \mathbf{U}$. Such choice functions violate prominent coherence constraints. Helzner articulates a sense in which "two-tiered choice functions emerge naturally from conditional choice" (2013, p. 946). In the context of conditional choice, two-tiered choice functions admit of a relatively simple characterization. This is not the case in the context of standard, unconditional choice functions, for which a characterization of such functions in terms of coherence properties is still unavailable (Arló-Costa, 2011). While the topic of two-tiered (or $n$-tiered) choice functions is important, it is not the focus of the present work. We are interested in the account of choice that results from conditional choice when only a single tier is relevant.

Just as admissibility generalizes the concept of preference, conditional admissibility generalizes the notion of admissibility. Helzner argues that the concept of admissibility as articulated above is insufficient for characterizing standards of synchronic rationality for individual decision makers. That is because an agent's judgments of admissibility depend on what the actual values of particular parameters are for that agent. Take expected utility for example. What the admissible options are for an agent depends, in part, on what her probabilities are at the time. But if an agent is synchronically committed to maximizing expected utility, this commitment, according to Helzner, should extend to conditional judgments about what would be admissible if her beliefs were somehow different, say, because she had received some piece of information other than the one she in fact received. Conditional choice functions are intended to encode information about the agent's conditional judgements of admissibility, information about the agent's judgments of admissibility given some epistemic or valuational state. For E-admissibility, admissibility in a menu can be thought of as determined by such conditional judgments - the judgments that an option is admissible relative to some probability function included in $\mathbf{P}$, an epistemic state different from $\mathbf{P}$ itself. But, on Helzner's view, an agent might hold fixed her commitment to $E$-admissibility and consider the decision problem under the supposition of any alternative credal state $\mathbf{P}^{\prime}$ such that $\mathbf{P}^{\prime} \neq \mathbf{P}$. The point, however, is not just that it is nice to also encode conditional judgments of admissibility for generality's sake. These judgements, he argues, can be gainfully employed. The next two motivations for conditional choice make use of conditional judgments of admissibility. 
In another paper, Helzner provides further motivation for conditional choice that is rooted firmly in the pragmatist tradition in epistemology (2011). At the fountainhead of pragmatist epistemology is the belief-doubt model. Levi writes, "In my judgment, the belief-doubt model represents the greatest insight in the pragmatist tradition, and I have sought to preserve it in my own thinking" (1991, p. 163). Peirce sketches the model in "The Fixation of Belief":

The irritation of doubt is the only immediate motive for the struggle to attain belief. It is certainly best for us that our beliefs should be such as may truly guide our actions so as to satisfy our desires; and this reflection will make us reject every belief which does not seem to have been so formed as to insure this result. But it will only do so by creating a doubt in the place of that belief. With the doubt, therefore, the struggle begins, and with the cessation of doubt it ends. (1992, p. 114)

In what sense is doubt an irritant on this view? It is not that doubt is merely psychologically uncomfortable, but that it interferes with action (Misak, 2004). Helzner suggests that, in the context of an admissibility account of rational decision making, one way of interpreting the claim that doubt interferes with rational action is that certain epistemic states not free from doubt lead to the violation of coherence constraints like $(I V)$ or even $(I I)$. For example, in the bet on Ellsberg's urn discussed above, uncertainty about the distribution of the urn resulted in violations of both $(I V)$ and $(I I)$. But on the belief-doubt model, Helzner argues, the agent needs to be aware of such violations if they are to prompt inquiry or an effort to eliminate them. And that requires conditional judgments of admissibility. The introduction of conditional judgments of admissibility provides for synchronically judging admissibility from states free from doubt as well as from states not free of doubt. Inquiring agents are thus in a position to assess the ways in which the relevant constraints are violated in the latter but not the former states.

A related, and perhaps more compelling, motivation for relativizing choice functions to states of information (or, more generally, states of commitment) concerns issues with the notion of the internal consistency of choice. The problem here is that violations of $\alpha$ and other internal consistency constraints can seem rational in certain fleshed-out contexts. Take the problem of the epistemic value of the menu (Sen, 1993). There is a very familiar example, due to Luce and Raiffa, that is supposed to demonstrate such a "rational" violation of $\alpha(1957$, p. 288). At a restaurant, a customer chooses salmon from a menu consisting of salmon and steak. Upon learning that fried snails and frog's legs are also options, however, the customer changes to steak. The explanation offered is that the customer initially fears that the restaurant would botch the steak, suspecting that the restaurant is not of good quality. The presence of the additional options on the menu, however, leads the customer to infer that the restaurant is a good one, and so she switches to steak. The menu carries information for the customer. Such choice behavior violates property $\alpha$. Steak is not judged admissible on the small menu despite being so judged on the large one.

The epistemic value of the menu has been addressed in various ways. One way to deflate the worry is to recognize that, in standard applications, choice functions depend on underlying epistemic or information states. The idea in outline is that in scenarios like the one above, the agent's state of information is not held fixed. 
Indeed, it is crucial to the example that the epistemic state changes. And if choice functions depend on states of information, the choice functions indexed by two distinct states of information will in general yield distinct choice sets. So we do not have an inconsistency between different parts of the same choice function in the restaurant example and the counterexample fails. In addressing other alleged counterexamples to internal consistency constraints, Sen employs a similar strategy by making choice functions dependent on the identity of the agent doing the choosing (1997). Arló-Costa explores the present strategy, and suggests framing the relevant epistemic states in terms of full beliefs and doxastic expectations (2006). ${ }^{8}$ In the restaurant example, the agent seems to assume by default that the restaurant is not good (or does not expect it to be good at any rate), with the larger menu occasioning a revision of her expectations. Helzner urges the present solution to the problem of the epistemic value of the menu (MS). He argues that confronting the problem requires a framework that allows for changes of epistemic states, and this goes beyond what the standard interpretation of set-valued choice functions is capable of. ${ }^{9}$

Let $\mathcal{E}=\langle E, \sqsubseteq\rangle$ be a non-empty partially ordered set such that every chain in $\mathcal{E}$ has an upper bound in $\mathcal{E}$. The elements of $E$ are intended to represent "the antecedent judgments upon which judgments of admissibility depend" (Helzner, 2013, p. 937). Helzner targets an interpretation in terms of information states, but notes that the relevant conditioning set might differ in different contexts. (The elements of $E$ might be sets of valuational states, or something more complex like sets of probability/utility pairs.) The $\sqsubseteq$ relation is meant to partially order the elements of $E$ according to their strength. $e \sqsubseteq f$ can then be read as " $f$ is at least as strong as e." If we were to represent information states as subsets of some possibility space, such as sets of possible worlds, it would be most natural to interpret $\sqsubseteq$ as reverse subset inclusion: $e \sqsubseteq f$ iff $f \subseteq e$. Similarly, reverse subset inclusion is a sensible interpretation of $\sqsubseteq$ when the elements of $E$ are interpreted as sets of probability functions $\grave{a}$ la indetermenate probabilities. If the information states are represented as deductively closed sets of sentences, subset inclusion makes sense, that is, $e \subseteq f$ iff $e \subseteq f$.

Definition 4. A function $\mathcal{C}: \mathcal{X} \times \mathcal{E} \rightarrow \mathscr{P}(X)$ is a conditional choice function just in case the following conditions are satisfied for all $x \in X, Y \in \mathcal{X}$ and $e \in E$ : (i) $\mathcal{C}(Y \mid e) \subseteq Y$ and (ii) if $x \in \mathcal{C}(Y \mid e)$, then there is an $f \in E$ such that $e \sqsubseteq f$ and $x \in \mathcal{C}(Y \mid g)$ whenever $f \sqsubseteq g .{ }^{10}$

Condition (i) simply requires that $\mathcal{C}$ be a choice function in its first argument. If the second argument is held fixed, a conditional choice function behaves like a regular choice function. Condition (ii) calls for a bit more explanation. Perhaps

\footnotetext{
${ }^{8}$ The sort of expectation intended here is the one of relevance to nonmonotonic logic and not the familiar sort of expectation of decision theory.

${ }^{9}$ This line of response to the epistemic value of the menu enjoys a serious advantage over a response of a different kind. Another way to respond is to insist that the option $w$ from menu $\{w, x\}$ is not the same as option $w$ from the menu $\{w, x, y, z\}$. Such a response does forestall counterexamples of the above sort, but at a rather severe cost. If an option changes depending on the other available options (e.g., steak at a bad restaurant versus steak at a good restaurant), it will be quite hopeless to attempt to invoke inter-menu consistency constraints. We risk emptying the theory of real content.

${ }^{10}$ Again, unlike Helzner, we do not make the more standard assumption that choice sets are non-empty.
} 
the most intuitive way to think about it is in terms of stability. If an option $x \in Y$ is admissible at $e$, then there is a way of filling out the information or strengthening $e$ such that $x$ becomes admissible and remains admissible regardless of any further strengthening of the information state. That is, there is some chain from $e$ on which the judgment of $x$ as admissible stabilizes. What (ii) demands is that only stably admissible options, in this sense, are admissible.

To take an example, suppose that the elements of $E$ are sets of possible worlds. Let $e=\left\{w_{1}, w_{2}\right\}$, so the agent believes that the real world is in $\left\{w_{1}, w_{2}\right\}$ (say, that a ball drawn from an urn is one of two possible colors), but cannot make further distinctions. According to (ii), the only acts admissible at $e$ are ones that are admissible at $\left\{w_{1}\right\}$ or $\left\{w_{2}\right\}$. If the elements of $E$ are convex sets of probabilities, condition (ii) demands that any option admissible given $\mathbf{P}$ must be stably admissible along some strengthening of $\mathbf{P}$.

Given a conditional choice function, we can recover a relation that represents a second-tier consideration. The basic idea is that some options on a menu $Y$ that might nonetheless be deemed admissible at stronger information states, might drop out of the choice set due to some second-tier cut. We define a binary relation $>_{e}$ on $X$.

Definition 5. Let $\mathcal{C}: \mathcal{X} \times \mathcal{E} \rightarrow \mathscr{P}(X)$ be a conditional choice function. $x>_{e} y$ iff there is $a Y \in \mathcal{X}$ and an $f \in E$ such that (i) $e \sqsubseteq f$, (ii) $x \in \mathcal{C}(Y \mid e)$, (iii) $y \notin \mathcal{C}(Y \mid e)$, and (iv) $y \in \mathcal{C}(Y \mid f)$.

Let $>_{e}^{t}$ be the transitive closure of $>_{e}$. Define $z_{e}^{t}$ by $x z_{e}^{t} y$ iff not $y>_{e}^{t} x$. Given some simple conditions, Helzner shows that we can characterize both maximization and optimization at the second tier with these relations (2013, pp. 944-945).

Consider again the betting setup above for Ellsberg's urn (Table 1). Both $f$ and $h$ are $E$-admissible relative to $\mathbf{P}$ in the menu $\{f, h\}$, but one could appeal to a secondary security condition in an attempt to distinguish between the $E$ admissible options. In that case, $h$, which has the best worst-case outcome, is uniquely admissible (the payoff is never less than $\frac{3}{2}$ whereas the lowest possible payoff for $f$ is 0$)$. But there is a strengthening of $\mathbf{P}, p$ such that $p($ Red $)=\frac{1}{3}$ and $p(B l u e)=\frac{2}{3}$, such that $f$ is uniquely admissible in $\{f, h\}$. So for a conditional choice function representing these judgments of admissibility, $h>_{\mathbf{P}} f$.

\section{Conditional Choice with a Vacuous Second Tier}

Sometimes the second (third, etc.) tier is vacuous. In such cases, the elements of $X$ are not distinguished with respect to the second tier. For instance, an agent or a collaborating group of agents might regard $E$-admissibility as a sufficient standard for admissibility, appealing to no secondary considerations. Or perhaps all of the options on a menu are equivalent with respect to, for example, security. Does conditional choice deliver a normatively adequate account under this restriction? Insofar as conditional choice is interesting in general, it is important to explore its structure. To the extent that single criterion decision-making is important, the class of conditional choice functions with vacuous second tiers is of special relevance. Paying attention to this class also positions us to better understand how Helzner's proposal generalizes the standard account of rational choice.

Like several recent studies, we have not insisted here that choice sets be nonempty (Seidenfeld et al, 2010; Arló-Costa and Pedersen, 2011; Arló-Costa, 2011). 
There may be reasons, however, for reservations about this particular generalization of the notion of a choice function. Rott defends empty choice sets by claiming that it may be that "every option on the menu appears entirely unacceptable" (his "taboo sets") (2001, p. 150). But if, in a particular decision problem, it is possible to refuse to choose among some alternatives, then, in one clear sense, one has the option to refuse to choose between those alternatives in that scenario. And if one refuses to make a selection from some set, that choice, the choice of abstaining from making a selection from some particular options, needs to be in some sense rationalized as optimal or shown to be admissible. What I am suggesting here is that "refusal to choose" might be more appropriately construed as just selecting a certain option on the menu (the option to not select one of the other items on the menu). If nothing from the menu is chosen, then the menu is not an accurate representation of the decision problem at hand - the agent selects the "abstain" option, which is not even on the menu. Fishburn, for example, addresses Rott's concern by insisting that some "abstain," "delay decision," or "maintain the status quo" option always be present, assuring some alternative must be selected (1973, p. 3).

Nevertheless, we have not made such assumptions about what counts as a decision menu in particular contexts of choice. A few tentative defenses can be offered for allowing empty choice sets. First, when it comes to extending choice functions to infinite menus, there may be no optimal or even maximal options. Consider an open set $Y=\{0 \leqslant x<1\}$ and a preference ordering that coincides with the ordering on $X$. Second, when an agent's admissibility-determining commitments are inconsistent (e.g., when an agent is certain both that it will rain and that it will not rain), all of the feasible options are ruled out as inconsistent with them. ${ }^{11}$ Third, in the background here is a project connecting constraints on choice functions to constraints on belief revision operators in the style of Rott. In that context, (Success) corresponds to a particular belief revision constraint. So, in the spirit of generality, we stick to this formulation of choice functions, noting that the relevant restrictions - namely, (Success) - might be desirable to impose after all.

As a result of allowing for empty choice sets, however, requiring that $>_{e}=\varnothing$ for all $e \in E$ does not by itself yield the right account of a vacuous second tier. Since no secondary considerations are invoked, at $e$ there are no grounds on which to rule out options deemed admissible at stronger states of commitment. Because $>_{e}=\varnothing$ when $C(Y \mid e)=\varnothing$ for all $Y \in \mathcal{X}$, in addition to requiring that $>_{e}=\varnothing$, we should insist that all options are not to be ruled out at $e$ (on the basis of what, after all?) if some option is admissible relative to some strengthening of $e$. We can formulate such a requirement as follows:

$$
\nu: \text { If } \mathcal{C}(Y \mid e)=\varnothing \text {, then } \mathcal{C}\left(Y \mid e^{\prime}\right)=\varnothing \text { for all } e^{\prime} \in E \text { such that } e \sqsubseteq e^{\prime} .
$$

$\nu$ is a reasonable constraint in contexts in which no secondary (tertiary, etc.) concerns are in play. It requires that weakening the state of admissibility-determining judgments does not rule out all options on any menu that has admissible options according to some stronger state of admissibility-determining judgments.

There is an equivalent formulation to these two requirements that makes a simple change to the second clause in the definition of conditional choice functions. The only difference is that condition (ii) is strengthened to an iff. Before, a judgment of conditional admissibility at an information state $e$ entailed stable admissibility

\footnotetext{
${ }^{11}$ I owe emphasizing this point to an anonymous referee.
} 
from $e$. The following condition makes those equivalent. Stable admissibility from $e$ entails conditional admissibility at $e$ too. Instead of $(i i)$, we have $\left(i i^{\prime}\right): x \in \mathcal{C}(Y \mid e)$ iff there is an $f \in E$ such that $e \sqsubseteq f$ and $x \in \mathcal{C}(Y \mid g)$ whenever $f \sqsubseteq g$. In the two-world information state example given above, conditional choice demanded stability of admissible options. What $\left(i i^{\prime}\right)$ adds is deeming any stably admissible option from $e$ as admissible at $e$. So, $\left(i i^{\prime}\right)$ adds that if an agent is in information state $\left\{w_{1}, w_{2}\right\}$, any options admissible at $\left\{w_{1}\right\}$ and any admissible at $\left\{w_{2}\right\}$ are admissible at $\left\{w_{1}, w_{2}\right\}$. We require that both ways of evaluating the options for admissibility are permissible.

Observation 1. Let $\mathcal{C}: \mathcal{X} \times \mathcal{E} \rightarrow \mathscr{P}(X)$ be a conditional choice function. $\mathcal{C}$ satisfies $\left(i i^{\prime}\right)$ iff $\mathcal{C}$ satisfies $\nu$ and $>_{e}=\varnothing$ for all $e \in E$.

Again, in the spirit of generality, we stick to Helzner's formulation of conditional choice. Instead of altering the definition of clause (ii), we express conditional choice with a vacuous second tier as conditional choice functions meeting the indicated conditions. Henceforward, conditional choice functions with vacuous second tiers will be denoted by $\tilde{\mathcal{C}}$.

A certain normative account of neutrality underwrites conditional choice, and is made explicit in the choice structure just described. That account has it that a neutral position with respect to conflicting judgments of a particular type preserves the shared agreements between the parties and introduces no judgments over which there is disagreement (Levi, 1986; Seidenfeld et al, 1989). This way, no relevant questions are begged and further deliberation or inquiry can be undertaken from such a perspective. As noted above, a primary motivation for the introduction of indeterminate probabilities is to provide a sufficiently neutral perspective that allows for the consideration of all the various rival theories, e.g., the statistical hypotheses consistent with the known data about the Ellsberg urn. Since the information about the urn does not rule out several possible distributions, the agent is not obliged to act as if a unique probability distribution is permissible in this case. We can think of a choice function as partitioning menus into the set of rejected options and its complement, the set of admissible options. How should we think of neutrality with respect to different judgments of admissibility? According to the view being presented, to suspend judgment between some number of rival ways of evaluating the options is to regard each of those ways as permissible for evaluating the options. Whatever the neutral position is with respect to the conditioning commitments (beliefs, values, etc.), judgments of admissibility relative to that state capture the shared agreements of all the strengthenings of that state in the following sense: all options deemed admissible at a strengthening of $e$ are admissible (not ruled out) at $e$ and any option that is inadmissible at every strengthening of $e$ remains so. ${ }^{12}$ On this view, judgments of admissibility at $e$ represent a consensus, or a neutral position, for the admissibility judgments at those stronger states.

\footnotetext{
${ }^{12}$ The "whatever the neutral position is" hedge is an important one. With it, I mean to avoid commiting to a particular position on a relevant and disputed philosophical issue. As mentioned earlier, Levi insists that credal states be convex: if $p_{1}, p_{2} \in \mathbf{P}$, then $\alpha p_{1}+(1-\alpha) p_{2} \in \mathbf{P}$ for $\alpha \in[0,1]$. The convex combination of two probability functions represents a compromise, and suspending judgment between two "rival" probability judgments, on his view, requires considering any compromise - and not just $p_{1}$ and $p_{2}$ - to be permissible to use in computing expectated utility. Levi imposes convexity on states of utility judgment as well. Focusing just on probability judgments, if we endorse convexity, the neutral position, $e$, for two distinct, determinate states of probability judgment, $p_{1}$ and $p_{2}$, will be the convex set generated by these two states. In that case,
} 
The following is easily derived from the conditional choice with a vacuous second tier structure and is perspicuous.

Observation 2. Let $\mathcal{C}: \mathcal{X} \times \mathcal{E} \rightarrow \mathscr{P}(X)$ be a conditional choice function with a vacuous second tier. For all $Y \in \mathcal{X}$ and $e \in E, \tilde{\mathcal{C}}(Y \mid e)=\bigcup\left\{\tilde{\mathcal{C}}\left(Y \mid e^{\prime}\right): e \sqsubseteq e^{\prime}\right\}$.

So at each information (or valuational, etc.) state, a conditional choice function with a vacuous second tier takes the union of the options deemed admissible at stronger information states. If an information state, $e$, represents neutrality among (or suspending judgment or doubt or open-mindedness about) information states higher up in the poset, $\tilde{\mathcal{C}}(Y \mid e)$ represents an analogous suspense of judgments of admissibility. As the state of information is strengthened, the agent can make finer discriminations and so, in general, will consider fewer options as admissible. Put another way, admissible options are those consistent with the agent's commitments; so as those commitments are strengthened, fewer options are in general consistent with them. As the state of admissibility-determining commitments is weakened, the agent keeps an open mind about the options deemed admissible at stronger information states. Such options are consistent with her commitments, so are not ruled out. The idea is to provide for a choice-theoretic analogue of neutrality or suspense of judgment. With the assumption that the second tier is vacuous, this choice structure makes clear that conditional choice endorses the normative account of neutrality above. Suspending judgment among some number of ways of evaluating the options is to regard each of those ways as permissible for evaluating the options.

Conditional choice with a vacuous second tier is also a formally fruitful choice structure. It is well-known that allowing for a second-tier complicates matters from a formal perspective. The construction under consideration is well-behaved in the sense of preserving important coherence constraints and generalizing both rationalizability and pseudo-rationalizability results, as explained in the following section. Relatedly, the structure that the assumption of a vacuous second tier preserves that the more general formulation of conditional choice abstracts away from allows us to maintain interesting connections to both the theory of belief revision and nonmonotonic logic. The topic of a companion article, versions of Rott's representation results in belief revision theory and nonmonotonic logic hold. Take belief revision theory. Briefly, Rott shows that for certain natural constructions of belief change operators in terms of choice functions, the coherence constraints for choice functions and the rationality postulates for belief change are in one-to-one correspondence.

the admissible options at $e$ are not just those admissible relative to $p_{1}$ and to $p_{2}$, but those options deemed admissible relative to any strengthening of the convex set, e. Similarly, only options ruled out at every strengthening of $e$-not just those ruled out at both $p_{1}$ and $p_{2}$-will be ruled out at $e$. This, according to Seidenfeld, et al., is an unwelcome consequence of convexity (2010). They insist that a neutral position with respect to two, distinct judgments of admissibility ought to preserve the shared agreements in admissibility judgments between those two states. For example, if $x \notin \tilde{\mathcal{C}}\left(Y \mid\left\{p_{1}\right\}\right)$ and $x \notin \tilde{\mathcal{C}}\left(Y \mid\left\{p_{2}\right\}\right)$, the inadmissibility of $x$ is a shared agreement that should be preserved at a neutral state, $e$. Such an agreement is not always preserved under convexity. With an adequate formulation of the conditioning set, $E,\left\{p_{1}, p_{2}\right\} \notin E$ if convexity is required of credal states. If convexity is relaxed, such a credal state can be legitimate. At issue here is whether we should seek consensus in judgments of admissibility or in reasons for judgments of admissibility (probability, utility, etc.) (Seidenfeld et al, 1989). Conditional judgments of admissibility might be seen as a way of walking a line between these two views, but in any case, we can deliver the right results for either view by suitably constraining $E$. 
So, for example, in constructing a contraction function in terms of a choice function, imposing important choice constraints on the choice function gives rise to important belief change constraints on the generated contraction operator ('soundness'). Going the other way, any contraction operator satisfying important belief change constraints can be represented as the rational choice-based contraction operator with the underlying choice function satisfying important choice constraints ('completeness'). Conditional choice with a vacuous second-tier allows for interesting versions of both directions of this representation result. I take this collection of results as evidence that conditional choice, far from emptying the standard account of content, is a fruitful generalization of that important theory.

\section{Connections to the Standard Theory of Choice Functions}

Finally, we consider some connections between conditional choice and the standard theory of rational choice. I include first a few of Helzner's observations that hold for conditional choice in general. ${ }^{13}$ Given a conditional choice function, we can generate an indexed family of standard choice functions by holding fixed the second argument. Let $\mathcal{C}_{e}: \mathcal{X} \rightarrow \mathscr{P}(X)$ be given by $\mathcal{C}_{e}(Y)=\mathcal{C}(Y \mid e)$ for all $Y \in \mathcal{X}$ and $e \in E$. We will refer to this family of choice functions as the local choice functions of $\mathcal{C}$.

Observation 3. If $\mathcal{C}: \mathcal{X} \times \mathcal{E} \rightarrow \mathscr{P}(X)$ is a conditional choice function on $X$ and $e \in E$, then $\mathcal{C}_{e}: \mathcal{X} \rightarrow \mathscr{P}(X)$ is a choice function on $X$.

Since Observation 3 holds for conditional choice functions in general, it holds for conditional choice functions with a vacuous second tier. Similarly, any choice function on $X$ can be viewed as a conditional choice function on $X$ with a vacuous second tier. It follows that any choice function can be viewed as a conditional choice function. Let $C^{\star}: \mathcal{X} \times \mathcal{E} \rightarrow \mathscr{P}(X)$ be given by $C^{\star}(Y \mid e)=C(Y)$ for all $e \in E$ and $Y \in \mathcal{X}$.

Observation 4. If $C$ is a choice function on $X$, then $C^{\star}$ is a conditional choice function on $X$ with a vacuous second tier.

Helzner also proposes a canonical way of extending criteria for choice functions to conditional choice criteria via the following translation.

Definition 6. Let $P$ be a constraint on choice functions. A conditional choice function $\mathcal{C}$ satisfies property $P^{\star}$ iff for every $e \in E$ there is an $f \in E$ such that $e \sqsubseteq f$ and $\mathcal{C}_{g}$ satisfies $P$ for all $g \in E$ such that $f \sqsubseteq g$.

Observation 5. Let $C$ be a choice function on $X$. Let $P$ be a property of choice functions. $C$ satisfies $P$ iff $C^{\star}$ satisfies $P^{\star}$.

The next observation is that if $\tilde{\mathcal{C}}$ satisfies $P^{\star}$, then, for all $Y \in \mathcal{X}$ and $e \in E, \tilde{\mathcal{C}}(Y \mid e)$ is the union of the choice sets of those local choice functions associated with $e^{\prime}$ such that $e \subseteq e^{\prime}$ that satisfy $P$.

Observation 6. Let $\tilde{\mathcal{C}}: \mathcal{X} \times \mathcal{E} \rightarrow \mathscr{P}(X)$ be a conditional choice function with a vacuous second tier and $P$ be a property of choice functions. If $\tilde{\mathcal{C}}$ satisfies $P^{\star}$, then $\tilde{\mathcal{C}}(Y \mid e)=\bigcup\left\{\tilde{\mathcal{C}}\left(Y \mid e^{\prime}\right): e \sqsubseteq e^{\prime}\right.$ and $\tilde{\mathcal{C}}_{e^{\prime}}$ satisfies $\left.P\right\}$ for all $Y \in \mathcal{X}$ and $e \in E$.

\footnotetext{
${ }^{13}$ The proofs of Observations 3-5 are Helzner's, with only a slight augmentation required for 4 (Helzner, 2013, pp. 940-941).
} 
Certain choice-functional constraints are preserved by taking the union of choice functions.

Observation 7. Properties $(I),\left(I^{-}\right),\left(I^{\prime}\right),(I I I),\left(I V^{+}\right),($Faith 1$),($ Faith 2$)$, (Success), $(\varnothing 1),(\varnothing 2)$, are each preserved under unions, i.e., if $\left\{C_{i}: i \in I\right\}$ is a collection of choice functions with $C_{i}: \mathcal{X} \rightarrow \mathscr{P}(X)$ satisfying one of these properties for all $i \in I$, then $C$ satisfies that property, where $C(Y)=\bigcup_{i \in I} C_{i}(Y)$ for all $Y \in \mathcal{X}$.

Conditional choice with a vacuous second tier is a more conservative extension of traditional rational choice theory. The following result, a consequence of Observations 6 and 7, does not hold for conditional choice functions in general and is one way of making this sense of conservativeness both clear and precise. ${ }^{14}$

Observation 8. Let $\tilde{\mathcal{C}}: \mathcal{X} \times \mathcal{E} \rightarrow \mathscr{P}(X)$ be a conditional choice function with a vacuous second tier, and let $\mathcal{X}$ be the set of all finite subsets of $X . \tilde{\mathcal{C}}$ satisfies $\left\{\begin{array}{c}\left(I^{\star}\right) \\ \left(I^{-\star}\right) \\ \left(I^{\prime \star}\right) \\ \left(I I I^{\star}\right) \\ \left(I V^{+\star}\right) \\ \left(\text { Faith } 1^{\star}\right) \\ \left(\text { Faith } 2^{\star}\right) \\ (\text { Success } \\ \left(\varnothing 1^{\star}\right) \\ \left(\varnothing 2^{\star}\right)\end{array}\right\}$ iff every local choice function of $\tilde{\mathcal{C}}$ satisfies $\left\{\begin{array}{c}(I) \\ \left(I^{-}\right) \\ \left(I^{\prime}\right) \\ (I I I) \\ \left(I V^{+}\right) \\ (\text {Faith1) } \\ (\text { Faith } 2) \\ (\text { Success }) \\ (\varnothing 1) \\ (\varnothing 2)\end{array}\right\}{ }^{15}$

This proposition says that imposing the conditional extension of certain choice constraints on a conditional choice function with a vacuous second tier implies that every local choice function satisfies the choice constraint itself. So, if the conditional choice function satisfies $\left(I^{\star}\right)$, then each of its local choice functions satisfies $(I)$. And if the conditional choice function satisfies $\left(I I I^{\star}\right)$, then each of its local choice functions satisfies $(I I I)$. Conversely, if every local choice function satisfies one of those properties, then $\tilde{\mathcal{C}}$ satisfies the conditional extension of that property.

Is there an interesting generalization of rationalizability for conditional choice functions? And which constraints, if any, characterize it? There is a very important absence from the lists of conditions in Observation 8. $\left(I I^{\star}\right)$ does not entail $(I I)$ in the above sense. To see this, consider the following example. Let $X=\{x, y, z\}$ and $\mathcal{X}=\mathscr{P}(X)$. Let $E=\left\{e, e^{\prime}, e^{\prime \prime}\right\}$ with $e \sqsubseteq e^{\prime}, e \sqsubseteq e^{\prime \prime}$, but $e^{\prime} \nsubseteq e^{\prime \prime}$ and $e^{\prime \prime} \nsubseteq e^{\prime}$. Assume that, at $e^{\prime}$, choice are made so as to optimize the weak order $z \geqslant x \geqslant y$, and at $e^{\prime \prime}$, choices are made so as to optimize the weak order $y \geqslant x \geqslant z$. This

\footnotetext{
${ }^{14}$ Consider the following counterexample for $(I)$, perhaps the most central constraint in the theory of rational choice. Let $\mathcal{C}$ be a conditional choice function, and let $X=\{x, y, z\}$ and $\mathcal{X}=\mathscr{P}(X)$. Let $E=\left\{e, e^{\prime}, e^{\prime \prime}\right\}$ with $e \sqsubseteq e^{\prime}, e \sqsubseteq e^{\prime \prime}$, but $e^{\prime} \nsubseteq e^{\prime \prime}$ and $e^{\prime \prime} \nsubseteq e^{\prime}$. Suppose that $\mathcal{C}_{e^{\prime}}$ and $\mathcal{C}_{e^{\prime \prime}}$ each satisfy $(I)$, and so $\tilde{\mathcal{C}}$ satisfies $\left(I^{\star}\right)$. Let $\mathcal{C}_{e^{\prime \prime}}$ optimize $z<y<x$. Let $\mathcal{C}_{e^{\prime}}$ optimize $x<y<z$. Let $\mathcal{C}(\{x, y, z\} \mid e)=\{x, z\}$ and $\mathcal{C}(\{x, y\} \mid e)=\{y\}$. It is clear that $\mathcal{C}_{e}$, while satisfying the definition of a conditional choice function, does not satisfy $(I)$. Compare Seidenfeld et al.'s Example 1 (2010, p. 158) and Helzner's Example 2 (2013, p. 932), both drawing heavily on Levi's work.

${ }^{15}$ Seidenfeld et al. characterize coherent choice with a set of four axioms on choice functions (2010). It turns out that an anologue of Observation 8 holds for those axioms. Because those axioms are rather different from the ones under consideration here, treating them will be left for future work.
} 
entails that $\tilde{\mathcal{C}}(\{x, y\} \mid e)=\{x, y\}, \tilde{\mathcal{C}}(\{x, z\} \mid e)=\{x, z\}$, and $\tilde{\mathcal{C}}(\{x, y, z\} \mid e)=\{y, z\}$. Since $x \in \tilde{\mathcal{C}}(\{x, y\} \mid e) \cap \tilde{\mathcal{C}}(\{x, z\} \mid e)$, but $x \notin \tilde{\mathcal{C}}(\{x, y, z\} \mid e), \tilde{\mathcal{C}}_{e}$ does not satisfy $(I I)$. By assumption, both $\tilde{\mathcal{C}}_{e^{\prime}}$ and $\tilde{\mathcal{C}}_{e^{\prime \prime}}$ do satisfy $(I I)$, hence guaranteeing that $\tilde{\mathcal{C}}$ satisfies $\left(I I^{\star}\right)$.

The reason that $(I I)$ 's absence is so important is that, as Theorem 1 says, $(I)$ and $(I I)$ are necessary and sufficient for rationalizability (assuming $(\varnothing 1)$ when we allow for empty choice sets). As the foregoing counterexample makes clear, $\left(I^{\star}\right)$ and $\left(I I^{\star}\right)$ do not secure the rationalizability of every local choice function of a conditional choice function with a vacuous second tier, $\tilde{\mathcal{C}}$. Is there a property, $\left(\rho^{\star}\right)$, of a conditional choice function that implies $(I I)$ for every local choice function? Here, I would like to urge a philosophical concern about any such $\left(\rho^{\star}\right)$. If every local choice function is to satisfy $(I I),\left(\rho^{\star}\right)$ would rule out the plainly sensible model above. $\tilde{\mathcal{C}}_{e^{\prime}}$ and $\tilde{\mathcal{C}}_{e^{\prime \prime}}$ are each rationalizable, but such a "disagreement" between them-just reversing the preferences of one another -is impermissible with $\left(\rho^{\star}\right)$ because $\tilde{\mathcal{C}}_{e}$ must then violate $(I I)$. Accordingly, $\left(\rho^{\star}\right)$ is too restrictive as an a priori constraint on conditional choice. Perhaps an intuitive first pass at an interesting generalization of rationalizability for conditional choice would be the rationalizability of every local choice function of $\tilde{\mathcal{C}}$. In my view, the foregoing considerations show that this route is not promising. Furthermore, in keeping with the accounts in decision theory that inspire conditional choice, it is not desirable to impose binariness on every choice function. The betting scenario above involving Ellsberg's urn serves as an example of when rational choice does not reduce to binary comparison between the options.

Helzner already has the key idea in his paper on conditional choice, but the stronger assumption of a vacuous second tier allows for correspondingly stronger rationalizability results. For each $e \in E$, define $O_{e}=\left\{R_{\tilde{\mathcal{C}}_{f}}: e \sqsubseteq f\right\}$. The members of $O_{e}$ are the revealed preference relations for the relevant local choice functions of $\tilde{\mathcal{C}}$. Say that $R \in O_{e}$ is rationalizing if the choice function from which it is generated is rationalizable by it; in symbols, $\tilde{\mathcal{C}}_{e^{\prime}}(Y)=\left\{x: x R_{\tilde{\mathcal{C}}_{e^{\prime}}} y\right.$ for all $\left.y \in Y\right\}$ for all $Y \in \mathcal{X}$. What the following observation says is that, for conditional choice functions with a vacuous second tier, satisfying $\left(I^{\star}\right)$ and $\left(I I^{\star}\right)$ is equivalent to its being conditionally rationalizable in the sense that, for any information state, $e$, the admissible options are those options that are admissible according to the rationalizing relations in $O_{e}$. Another way to look at conditional rationalizability is that $\tilde{\mathcal{C}}(Y \mid e)$ is the set of options that are admissible at the relevant rationalizable or normal local choice functions of $\tilde{\mathcal{C}}$-relevant in the sense that $\tilde{\mathcal{C}}_{e^{\prime}}$ is relevant if $e \sqsubseteq e^{\prime}$.

Observation 9. Let $\tilde{\mathcal{C}}: \mathcal{X} \times \mathcal{E} \rightarrow \mathscr{P}(X)$ be a conditional choice function with a vacuous second tier satisfying $\left(\varnothing 1^{\star}\right)$ and let $\mathcal{X}$ be all the finite subsets of $X . \tilde{\mathcal{C}}$ satisfies $\left(I^{\star}\right)$ and $\left(I I^{\star}\right)$ iff $\tilde{\mathcal{C}}(Y \mid e)=\{x: x R y$ for all $y \in Y$ with respect to some rationalizing $\left.R \in O_{e}\right\}$ for all $Y \in \mathcal{X}$ and $e \in E$.

Instead of rationalizability by a single binary relation, conditional rationalizability is rationalizability by a set of such relations. Similarly, for rationalizability by a set of weak orders instead of by a single one, we have the following (the proof is analogous to that of Observation 9 and is omitted).

Observation 10. Let $\tilde{\mathcal{C}}: \mathcal{X} \times \mathcal{E} \rightarrow \mathscr{P}(X)$ be a conditional choice function with a vacuous second tier satisfying $\left(\varnothing 1^{\star}\right)$ and let $\mathcal{X}$ be all the finite subsets of $X . \tilde{\mathcal{C}}$ 
satisfies $\left(I^{\star}\right)$ and $\left(I V^{\star}\right)$ iff $\tilde{\mathcal{C}}(Y \mid e)=\{x: x R y$ for all $y \in Y$ with respect to some weak order $\left.R \in O_{e}\right\}$ for all $Y \in \mathcal{X}$ and $e \in E .^{16}$

It may be clear that conditional rationalizability and pseudo-rationalizability are closely related, but how exactly? First, the collection of choice functions that pseudo-rationalize a choice function must themselves be weak order rationalizable. As Observation 9 shows, conditional choice allows for a characterization of a generalization of pseudo-rationalizability in that the choice functions need to be merely rationalizable instead of weak order rationalizable. If $\tilde{\mathcal{C}}$ satisfies $\left(I^{\star}\right)$ and $\left(I I^{\star}\right)$, still some of its local choice functions might not satisfy both $(I)$ and $(I I)$ (some might not satisfy $(I I)$, specifically). But each local choice function is conditionally rationalizable by exactly those local choice functions associated with information states at least as strong as the one associated with the local choice function being rationalized that do satisfy both $(I)$ and $(I I)$. That is, at every local choice function, $\tilde{\mathcal{C}}_{e}$, admissibility on a menu is determined by the local choice functions, $\tilde{\mathcal{C}}_{e^{\prime}}$, such that $e \sqsubseteq e^{\prime}$ and $\tilde{\mathcal{C}}_{e^{\prime}}$ is rationalizable.

Second, cases in which $\tilde{\mathcal{C}}$ satisfies both $\left(I^{\star}\right)$ and $\left(I V^{\star}\right)$, conditional rationalizability by a set of weak orders amounts to the pseudo-rationalizability of every local choice function, $\tilde{\mathcal{C}}_{e}$, by the weak order rationalizable local choice functions associated with $e^{\prime} \in E$ such that $e \sqsubseteq e^{\prime}$.

Observation 11. Let $\tilde{\mathcal{C}}: \mathcal{X} \times \mathcal{E} \rightarrow \mathscr{P}(X)$ be a conditional choice function with a vacuous second tier satisfying $\left(\varnothing 1^{\star}\right)$ and let $\mathcal{X}$ be all the finite subsets of $X$. $\tilde{\mathcal{C}}$ satisfies $\left(I^{\star}\right)$ and $\left(I V^{\star}\right)$ iff $\tilde{\mathcal{C}}_{e}$ is pseudo-rationalizable by the set of local choice functions $\left\{\tilde{\mathcal{C}}_{e}^{\prime}: e \sqsubseteq e^{\prime}\right.$ and $\tilde{\mathcal{C}}_{e^{\prime}}$ is weak order rationalizable $\}$ for all $e \in E$.

Here, I have abused terminology somewhat. If we want to mirror Aizerman and Malishevski's result more closely, we can consider the case in which the set $X$ of options is finite. We have that, for all $Y \in \mathcal{X}$ and $e \in E, \tilde{\mathcal{C}}_{e}(Y)=\bigcup\left\{\tilde{\mathcal{C}}_{e^{\prime}}(Y): e \sqsubseteq e^{\prime}\right\}$, where $\tilde{\mathcal{C}}_{e^{\prime}}$ is weak order rationalizable. To see that we can appeal to just a finite set of local choice functions to do the pseudo-rationalizing, it suffices to observe that because the set of options is finite, there are only finitely many weak orderings possible. $X$ is finite so $X \times X$ is finite. Then, $\mathscr{P}(X \times X)$ must be finite, too. But any weak ordering of $X$ is some member of $\mathscr{P}(X \times X)$.

Observation 11 is potentially interesting because, within the conditional choice framework, the basic idea of pseudo-rationalization can be characterized by different constraints (namely, the conditional extensions of the most familiar constraints!). This alternative characterization might give us some insight into both how indeterminacy enters the choice function framework, and how conditional choice generalizes the standard choice function framework.

\section{Conclusion}

Not always will a second tier decision criterion be relevant. Sometimes, representing an agent's or a group's commitments requires no appeal to a second (or third, etc.) type of criterion. Other times, the second tier might be vacuous because the options are equivalent with respect to the secondary criterion. This paper studies the properties of the class of conditional choice functions with a vacuous

\footnotetext{
${ }^{16}$ Helzner shows instead that $\mathcal{C}$ satisfies $\left(I^{\star}\right)$ and $\left(I V^{\star}\right)$ iff $\mathcal{C}(Y \mid e) \subseteq\{x: x R y$ for all $y \in Y$ wrt some weak order $\left.R \in O_{e}\right\}$.
} 
second tier. It is argued that the resulting account is normatively well-motivated. That motivation comes from a conception of what it is to be neutral or in suspense with respect to a certain set of judgments.

The standard (weak order) rationalizability assumptions for choice functions have been called into question for a number of reasons by researchers in different fields. These reasons include concerns about the notion of the internal consistency of choice, issues about how to understand uncertainty, the possibility of conflicts in values, and the extension of the choice function framework to social contexts. Naturally, various formalisms have been explored. Concerns having to do with uncertainty have helped to guide the development of conditional choice. The potential to address certain puzzles about the concept of the internal coherence of choice counts as motivation for the conditional choice construction, too. Pseudorationalizability can be interpreted as a rationality constraint in cases in which there is indeterminacy in beliefs or values. Pseudo-rationalizability also has interesting interpretations in social choice contexts. For instance, a pseudo-rationalizable choice function can be understood as a social choice function with the relevant collection of weak order rationalizable choice functions representing voters or different evaluational criteria. We have seen how this concept relates to the rationalization of a conditional choice function. The motivations and results above hopefully recommend further consideration of conditional choice. 


\section{Appendix: Proofs}

\section{Proof of Observation 1}

Proof. Let $\mathcal{C}: \mathcal{X} \times \mathcal{E} \rightarrow \mathscr{P}(X)$ be a conditional choice function.

$(\Leftarrow)$ Suppose that $\mathcal{C}$ satisfies $\nu$ and that $>_{e}=\varnothing$ for all $e \in E$. Let $x \in \mathcal{C}(Y \mid e)$. Since $\mathcal{C}$ is a conditional choice function, it follows immediately that there is an $f \in E$ such that $e \subseteq f$ and $x \in \mathcal{C}(Y \mid g)$ whenever $f \sqsubseteq g$. For the other direction of $\left(i i^{\prime}\right)$, assume that there is an $f \in E$ such that $e \sqsubseteq f$ and $x \in \mathcal{C}(Y \mid g)$ whenever $f \sqsubseteq g$. By $\nu$, we have $\mathcal{C}(Y \mid e) \neq \varnothing$. So there is some $y \in \mathcal{C}(Y \mid e)$. Suppose that $x \notin \mathcal{C}(Y \mid e)$. It follows that $y>_{e} x$, which is a contradiction. So $x \in \mathcal{C}(Y \mid e)$. Hence, $\mathcal{C}$ satisfies $\left(i i^{\prime}\right)$.

$(\Rightarrow)$ Suppose that $\mathcal{C}$ satisfies $\left(i i^{\prime}\right)$. Suppose that $\mathcal{C}\left(Y \mid e^{\prime}\right) \neq \varnothing$ for some $e^{\prime} \in E$ such that $e \sqsubseteq e^{\prime}$. Then there is some $x \in \mathcal{C}\left(Y \mid e^{\prime}\right)$. By $\left(i i^{\prime}\right)$, there is an $f \in E$ such that $e^{\prime} \sqsubseteq f$ and $x \in \mathcal{C}(Y \mid g)$ whenever $f \sqsubseteq g$. But $e \sqsubseteq f$ too, so by $\left(i i^{\prime}\right), x \in \mathcal{C}(Y \mid e)$, so $\mathcal{C}(Y \mid e) \neq \varnothing$. Hence, $\mathcal{C}$ satisfies $\nu$. Now suppose that $>_{e} \neq \varnothing$ for some $e \in E$. Then, for some $Y \in \mathcal{X}$, there are $x, y \in Y$ such that $x \in \mathcal{C}(Y \mid e), y \notin \mathcal{C}(Y \mid e)$, but $y \in \mathcal{C}(Y \mid f)$ for some $f \in E$ such that $e \sqsubseteq f$. It follows from $\left(i i^{\prime}\right)$ that there is an $f^{\prime} \in E$ such that $f \sqsubseteq f^{\prime}$ and $y \in \mathcal{C}(Y \mid g)$ whenever $f^{\prime} \sqsubseteq g$. But since $e \sqsubseteq f^{\prime}$, by $\left(i i^{\prime}\right), y \in \mathcal{C}(Y \mid e)$, which is a contradiction. Thus, $>_{e}=\varnothing$ for all $e \in E$.

\section{Proof of ObSERVATION 2}

Proof. That $\tilde{\mathcal{C}}(Y \mid e) \subseteq \bigcup\left\{\tilde{\mathcal{C}}\left(Y \mid e^{\prime}\right): e \sqsubseteq e^{\prime}\right\}$ holds for all $Y \in \mathcal{X}$ and $e \in E$ is easy to see from the fact that $e \subseteq e$. For the other inclusion claim, let $x$ be in $\bigcup\left\{\tilde{\mathcal{C}}\left(Y \mid e^{\prime}\right): e \sqsubseteq\right.$ $\left.e^{\prime}\right\}$. By $\nu, \tilde{\mathcal{C}}(Y \mid e) \neq \varnothing$. So there is some $y \in \tilde{\mathcal{C}}(Y \mid e)$. Suppose that $x$ is not in $\tilde{\mathcal{C}}(Y \mid e)$. It follows that $y>_{e} x$, which contradicts the assumption that the second tier is vacuous. Hence, $x \in \tilde{\mathcal{C}}(Y \mid e)$.

\section{Proof of ObSERVATION 3}

Proof. Immediate from condition (i) in the definition of conditional choice, since a conditional choice function is a choice function in its first argument.

\section{Proof of Observation 4}

Proof. Let $C$ be a choice function on $X . C^{\star}: \mathcal{X} \times \mathcal{E} \rightarrow \mathscr{P}(X)$ since, for all $Y \in \mathcal{X}$ and $e \in E, C^{\star}(Y \mid e)=C(Y)$. For condition (i), $C^{\star}(Y \mid e) \subseteq Y$ because $C(Y) \subseteq Y$ for all $Y \in \mathcal{X}$. As for condition (ii), first suppose that $x \in C^{\star}(Y \mid e)$. Since, for any $e^{\prime} \in E, C^{\star}(Y \mid e)=C^{\star}\left(Y \mid e^{\prime}\right)$, it follows that there is an $f \in E$ such that $e \sqsubseteq f$ and $x \in C^{\star}(Y \mid g)$ whenever $f \sqsubseteq g$. Finally, for the other direction, suppose that there is an $f \in E$ such that $e \sqsubseteq f$ and $x \in C^{\star}(Y \mid g)$ whenever $f \sqsubseteq g$. Again, since $C^{\star}(Y \mid f)=C(Y)=C^{\star}\left(Y \mid e^{\prime}\right)$ for all $e^{\prime} \in E$, it follows that $x \in C^{\star}(Y \mid e)$.

\section{Proof of Observation 5}

Proof. Suppose that $C$ is a choice function on $X$ and satisfies $P$. Since $C_{e}^{\star}=C$ for all $e \in E$, it follows that $C^{\star}$ satisfies $P^{\star}$. For the other direction, assume that $C^{\star}$ satisfies $P^{\star}$. Let $e \in E$. By assumption, we have it that there is an $f \in E$ such that $e \sqsubseteq f$ and, for all $g \in E$ such that $f \sqsubseteq g, C_{g}^{\star}$ satisfies $P$. But this implies that $C_{f}^{\star}$ satisfies $P$ and $C_{f}^{\star}=C$, so $C$ satisfies $P$. 


\section{Proof of Observation 6}

Proof. Suppose that $\tilde{\mathcal{C}}$ satisfies $P^{\star}$ and that $x \in \tilde{\mathcal{C}}(Y \mid e)$. By definition, there is some $f \in E$ such that $e \sqsubseteq f$ and $x \in \tilde{\mathcal{C}}(Y \mid g)$ when $f \sqsubseteq g$. From the assumption, we have that there is some $f^{\prime} \in E$ such that $f \sqsubseteq f^{\prime}$ and $\tilde{\mathcal{C}}_{g^{\prime}}$ satisfies $P$ whenever $f^{\prime} \sqsubseteq g^{\prime}$. It follows that $x \in \bigcup\left\{\tilde{\mathcal{C}}\left(Y \mid e^{\prime}\right): e \sqsubseteq e^{\prime}\right.$ and $\tilde{\mathcal{C}}_{e^{\prime}}$ satisfies $\left.P\right\}$. Now assume that $x \in \bigcup\left\{\tilde{\mathcal{C}}\left(Y \mid e^{\prime}\right): e \sqsubseteq e^{\prime}\right.$ and $\tilde{\mathcal{C}}_{e^{\prime}}$ satisfies $\left.P\right\}$. It follows immediately from Observation 2 that $x \in \tilde{\mathcal{C}}(Y \mid e)$.

\section{Proof of Observation 7}

Proof. Let $\left\{C_{i}: i \in I\right\}$ be a collection of choice functions, $C_{i}: \mathcal{X} \rightarrow \mathscr{P}(X)$, and define $C(Y)=\bigcup_{i \in I} C_{i}(Y)$, for all $Y \in \mathcal{X}$. It is clear that $C$ is a well-defined choice function. We check each axiom.

$(I)$. Assume that for all $i \in I, C_{i}$ satisfies $(I)$. Suppose that $Y \subseteq Y^{\prime}$ and $x \in Y \cap C\left(Y^{\prime}\right)$. Since $x \in C\left(Y^{\prime}\right)$, it follows that $x \in C_{i}\left(Y^{\prime}\right)$ for some $i \in I$. By assumption $C_{i}$ satisfies $(I)$, so it follows that $x \in C_{i}(Y)$. Hence, $x \in C(Y)$, too. So, $C$ satisfies $(I)$.

$\left(I^{-}\right)$. Suppose that for all $i \in I, C_{i}$ satisfies $\left(I^{-}\right)$. Assume that $Y \subseteq Y^{\prime}$, $C\left(Y^{\prime}\right) \subseteq Y$, and $x \in C\left(Y^{\prime}\right)$. Then, for some $i \in I, x \in C_{i}\left(Y^{\prime}\right)$. Since $C_{i}$ satisfies $\left(I^{-}\right)$, it follows that $x \in C_{i}(Y)$. Hence, $x \in C(Y)$. So, $C$ satisfies $\left(I^{-}\right)$.

$\left(I^{\prime}\right)$. Suppose that $C_{i}$ satisfies $\left(I^{\prime}\right)$ for all $i \in I$. Assume that $x \in C\left(Y \cup Y^{\prime}\right)$. So, for some $i \in I, x \in C_{i}\left(Y \cup Y^{\prime}\right)$. By $\left(I^{\prime}\right), x \in\left[C_{i}(Y) \cup C_{i}\left(y^{\prime}\right)\right]$. In either case, it follows that $x \in\left[C(Y) \cup C\left(Y^{\prime}\right)\right]$. So $C$ satisfies $\left(I^{\prime}\right)$.

$(I I I)$. Let $C_{i}$ satisfy $(I I I)$ for all $i \in I$. Suppose that $Y \subseteq Y^{\prime}$ and $C\left(Y^{\prime}\right) \subseteq Y$, and let $x$ be in $C(Y)$. It must then be the case that $C_{i}\left(Y^{\prime}\right) \subseteq Y$ for all $i \in I$. And, for some $i \in I, x \in C_{i}(Y)$. By $(I I I), x \in C_{i}\left(Y^{\prime}\right)$. Hence, $x \in C\left(Y^{\prime}\right)$. It follows that $C$ satisfies $(I I I)$.

$\left(I V^{+}\right)$. Let $C_{i}$ satisfy $\left(I V^{+}\right)$for all $i \in I$. Assume that $Y \subseteq Y^{\prime}$ and $x \in C(Y)$. It follows that $x \in C_{i}(Y)$ for some $i \in I$. By $\left(I V^{+}\right), x \in C_{i}\left(Y^{\prime}\right)$. Thus, $x \in C\left(Y^{\prime}\right)$. $C$ therefore satisfies $\left(I V^{+}\right)$.

(Faith1). Suppose that (Faith1) is satisfied by $C_{i}$ for all $i \in I$. Assume that $Y \cap B \neq \varnothing$ (where $B$ is the set of "absolutely satisfactory" options in $X$ ) and let $x$ be in $C(Y)$. It follows that there is an $i \in I$ such that $x \in C_{i}(Y)$. By (Faith1), $x \in B$. So, $C$ satisfies (Faith1).

(Faith2). Let $C_{i}$ satisfy (Faith2) for all $i \in I$. Assume that $x \in Y \cap B$. By (Faith2), $x \in C_{i}(Y)$ for all $i \in I$. Hence, $x \in C(Y)$. $C$ therefore satisfies (Faith2).

(Success). Suppose that $C_{i}$ satisfies (Success) for all $i \in I$. Consider some $Y \neq \varnothing$ in $\mathcal{X}$. By (Success), $C_{i}(Y) \neq \varnothing$ for all $i \in I$. Hence, $C(Y) \neq \varnothing$. So, $C$ satisfies (Success).

$(\varnothing 1)$. Let $C_{i}$ satisfy $(\varnothing 1)$ for all $i \in I$. Suppose that $Y \subseteq Y^{\prime}$ and that $C\left(Y^{\prime}\right)=$ $\varnothing$. Then, $C_{i}\left(Y^{\prime}\right)=\varnothing$ for all $i \in I$. By $(\varnothing 1)$, it follows that $C_{i}(Y)=\varnothing$ for all $i \in I$. Hence, $C(Y)=\varnothing$, too. So, $C$ satisfies $(\varnothing 1)$.

$(\varnothing 2)$. Suppose that $C_{i}$ satisfies $(\varnothing 2)$ for all $i \in I$. Assume that $Y \subseteq Y^{\prime}$ and $C(Y)=\varnothing$. Then, $C_{i}(Y)=\varnothing$ for all $i \in I$. By $(\varnothing 2)$, we have that $C_{i}\left(Y^{\prime}\right) \cap Y=\varnothing$ for all $i \in I$. It follows that $C\left(Y^{\prime}\right) \cap Y=\varnothing$. $C$ therefore satisfies $(\varnothing 2)$. 


\section{Proof of Observation 8}

Proof. $(\Leftarrow)$ Since, by hypothesis, every local choice function of $\tilde{\mathcal{C}}$ satisfies property $P$, it follows that, for every $e \in E$ there is an $f \in E$ such that $e \sqsubseteq f$ and $\tilde{\mathcal{C}}_{g}$ satisfies $P$ for all $g \in E$ such that $f \sqsubseteq g$. That is, $\tilde{\mathcal{C}}$ satisfies $P^{\star}$.

$(\Rightarrow)$ Let $\tilde{\mathcal{C}}$ satisfy $P^{\star}$. By Observation $6, \tilde{\mathcal{C}}(Y \mid e)=\bigcup\left\{\tilde{\mathcal{C}}\left(Y \mid e^{\prime}\right): e \sqsubseteq e^{\prime}\right.$ and $\tilde{\mathcal{C}}_{e^{\prime}}$ satisfies $\left.P\right\}$ for all $Y \in \mathcal{X}$ and $e \in E$. For those constraints, $P$, that are preserved under unions (Observation 7 ), it follows that $\tilde{\mathcal{C}}_{e}$ satisfies $P$. Hence, every local choice function of $\tilde{\mathcal{C}}$ satisfies $P$.

\section{Proof of Observation 9}

Proof. Assume that $\tilde{\mathcal{C}}: \mathcal{X} \times \mathcal{E} \rightarrow \mathscr{P}(X)$ is a conditional choice with a vacuous second tier that satisfies $\left(\varnothing 1^{\star}\right)$, and let $\mathcal{X}=\mathscr{P}_{\text {fin }}(X)$.

$\Leftrightarrow$ Suppose that $\tilde{\mathcal{C}}$ satisfies $\left(I^{\star}\right)$ and $\left(I I^{\star}\right)$. By Observation 8, every local choice function of $\tilde{\mathcal{C}}$ satisfies $(\varnothing 1)$ and $(I)$. From Observation 6 it follows that $\tilde{\mathcal{C}}(Y \mid e)=\bigcup\left\{\tilde{\mathcal{C}}\left(Y \mid e^{\prime}\right): e \sqsubseteq e^{\prime}\right.$ and $\tilde{\mathcal{C}}_{e^{\prime}}$ satisfies $\left.(I I)\right\}$ for all $Y \in \mathcal{X}$ and $e \in E$. So, $\tilde{\mathcal{C}}(Y \mid e)=\bigcup\left\{\tilde{\mathcal{C}}\left(Y \mid e^{\prime}\right): e \sqsubseteq e^{\prime}\right.$ and $\tilde{\mathcal{C}}_{e^{\prime}}$ satisfies $(\varnothing 1),(I)$, and $\left.(I I)\right\}$. By Theorem 1, $\tilde{\mathcal{C}}(Y \mid e)=\bigcup\left\{\tilde{\mathcal{C}}\left(Y \mid e^{\prime}\right): e \sqsubseteq e^{\prime}\right.$ and $\tilde{\mathcal{C}}_{e^{\prime}}$ is rationalizable $\}=\{x: x R y$ for all $y \in Y$ with respect to some rationalizing $\left.R \in O_{e}\right\}$.

$(\Leftarrow)$ Suppose that, for all $x \in X, Y \in \mathcal{X}$, and $e \in \mathcal{E}, \tilde{\mathcal{C}}(Y \mid e)=\{x: x R y$ for all $y \in Y$ wrt some rationalizing $\left.R \in O_{e}\right\}$. Let $\mathcal{E}_{e}$ be the poset $\langle\{f \mid e \sqsubseteq f\}$, ㄱ, where $\subseteq$ is the relevant restriction of the partial order from $\mathcal{E}$. By the assumption on $\mathcal{E}$, it follows that every chain in $\mathcal{E}_{e}$ has an upper bound in $\mathcal{E}_{e}$. Thus, by Zorn's lemma, $\mathcal{E}_{e}$ has a maximal element. Let $f$ be such a maximal element of $\mathcal{E}_{e}$. Either (i) $\tilde{\mathcal{C}}(Y \mid f) \neq \varnothing$ for some $Y \in \mathcal{X}$ or (ii) $\tilde{\mathcal{C}}_{f}$ always returns the empty set. Suppose that (i). Then, for some $Y \in \mathcal{X}$, there is some $x \in \tilde{\mathcal{C}}(Y \mid f)$. By assumption, there is a rationalizing $R \in O_{f}$ such that $x R y$ for all $y \in Y$. $f$ is maximal so $R_{\tilde{\mathcal{C}}_{f}}$ is rationalizing. Therefore, by Theorem $1, \tilde{\mathcal{C}}_{f}$ satisfies $(I)$ and $(I I)$. And because $f$ is maximal, $\tilde{\mathcal{C}}_{g}$ satisfies $(I)$ and $(I I)$ for any $g \in E$ such that $f \sqsubseteq g$. Now, consider case (ii). $\tilde{\mathcal{C}}(Y \mid f)=\varnothing$ for all $Y \in \mathcal{X}$. It is straightforward to verify that $\tilde{\mathcal{C}}_{f}$ vacuously satisfies constraints $(I)$ and $(I I)$. Again, because $f$ is maximal, $\tilde{\mathcal{C}}_{g}$ satisfies $(I)$ and $(I I)$ for any $g \in E$ such that $f \sqsubseteq g$. It follows that $\tilde{\mathcal{C}}$ satisfies $\left(I^{\star}\right)$ and $\left(I I^{\star}\right)$.

\section{Proof of OBSERVATION 11}

Proof. Assume that $\tilde{\mathcal{C}}: \mathcal{X} \times \mathcal{E} \rightarrow \mathscr{P}(X)$ is a conditional choice with a vacuous second tier that satisfies $\left(\varnothing 1^{\star}\right)$, and let $\mathcal{X}=\mathscr{P}_{\text {fin }}(X)$.

$(\Leftrightarrow)$ Assume that $\tilde{\mathcal{C}}$ satisfies $\left(I^{\star}\right)$ and $\left(I V^{\star}\right)$. By Observation 8, it follows from our assumptions that $\tilde{\mathcal{C}}$ satisfies $(\varnothing 1)$ and $(I)$ at each of its local choice functions. By Observation 6, $\tilde{\mathcal{C}}(Y \mid e)=\bigcup\left\{\tilde{\mathcal{C}}\left(Y \mid e^{\prime}\right): e \sqsubseteq e^{\prime}\right.$ and $\tilde{\mathcal{C}}_{e^{\prime}}$ satisfies $\left.(I V)\right\}$ for all $Y \in \mathcal{X}$ and $e \in E$. Putting these together, it follows that $\tilde{\mathcal{C}}(Y \mid e)=\bigcup\left\{\tilde{\mathcal{C}}\left(Y \mid e^{\prime}\right): e \sqsubseteq e^{\prime}\right.$ and $\tilde{\mathcal{C}}_{e^{\prime}}$ satisfies $(\varnothing 1),(I)$, and $\left.(I V)\right\}$ for all $Y \in \mathcal{X}$ and $e \in E$. By Theorem 2, this amounts to $\tilde{\mathcal{C}}(Y \mid e)=\bigcup\left\{\tilde{\mathcal{C}}\left(Y \mid e^{\prime}\right): e \sqsubseteq e^{\prime}\right.$ and $\tilde{\mathcal{C}}_{e^{\prime}}$ is weak order rationalizable $\}$ for all $Y \in \mathcal{X}$ and $e \in E$.

$(\Leftarrow)$ Now assume that for all $e \in E, \tilde{\mathcal{C}}_{e}$ is pseudo-rationalizable by a collection of local choice functions $\left\{\tilde{\mathcal{C}}_{e^{\prime}}: e \sqsubseteq e^{\prime}\right.$ and $\tilde{\mathcal{C}}_{e^{\prime}}$ is weak order rationalizable $\}$. We establish that $\tilde{\mathcal{C}}$ satisfies $\left(I^{\star}\right)$ and $\left(I V^{\star}\right)$. Let $\mathcal{E}_{e}$ be the poset $\langle\{f \mid e \sqsubseteq f\}$, $\sqsubseteq\rangle$, where $\sqsubseteq$ is the relevant restriction of the partial order from $\mathcal{E}$. By the assumption on $\mathcal{E}$, it follows 
that every chain in $\mathcal{E}_{e}$ has an upper bound in $\mathcal{E}_{e}$. Thus, by Zorn's lemma, $\mathcal{E}_{e}$ has a maximal element. Let $f$ be such a maximal element of $\mathcal{E}_{e}$. Either (i) $\tilde{\mathcal{C}}(Y \mid f) \neq \varnothing$ for some $Y \in \mathcal{X}$ or (ii) $\tilde{\mathcal{C}}_{f}$ always returns the empty set. Consider case $(i)$ first. Suppose that, for some $Y \in \mathcal{X}, x \in \tilde{\mathcal{C}}(Y \mid f)$. By the assumption of the pseudorationalizability of each local choice function (i.e., $\tilde{\mathcal{C}}(Y \mid e)=\bigcup\left\{\tilde{\mathcal{C}}\left(Y \mid e^{\prime}\right): e \sqsubseteq e^{\prime}\right.$ and $\tilde{\mathcal{C}}_{e^{\prime}}$ is weak order rationalizable $\}$ for all $Y \in \mathcal{X}$ and $e \in E$ ), it follows that there is a weak order rationalizable local choice function $\tilde{\mathcal{C}}_{f^{\prime}}$ such that $f \sqsubseteq f^{\prime}$ and $x \in \tilde{\mathcal{C}}_{f^{\prime}}(Y)$. $f$ is maximal so $\tilde{\mathcal{C}}_{f}$ must itself be weak order rationalizable. Therefore, by Theorem $2, \tilde{\mathcal{C}}_{f}$ satisfies $(I)$ and $(I V)$. And because $f$ is maximal, $\tilde{\mathcal{C}}_{g}$ satisfies $(I)$ and $(I V)$ for any $g \in E$ such that $f \sqsubseteq g$. Now consider case $(i i), \tilde{\mathcal{C}}_{f}(Y)=\varnothing$ for all $Y \in \mathcal{X}$. $(I)$ and $(I V)$ are trivially satisfied. Because $f$ is maximal, $\tilde{\mathcal{C}}_{g}$ satisfies $(I)$ and $(I V)$ for any $g \in E$ such that $f \sqsubseteq g$. It follows that $\tilde{\mathcal{C}}$ satisfies $\left(I^{\star}\right)$ and $\left(I V^{\star}\right)$.

\section{REFERENCES}

Aizerman M, Malishevski A (1981) General theory of best variants choice: Some aspects. Automatic Control, IEEE Transactions on 26(5):1030-1040

Arló-Costa H (2006) Rationality and value: The epistemological role of indeterminate and agent-dependent values. Philosophical Studies 128(1):7-48

Arló-Costa H (2011) Indeterminacy and belief change. In: Dynamic Formal Epistemology, Springer, pp 173-195

Arló-Costa H, Pedersen AP (2011) Social norms, rational choice and belief change. Belief revision meets philosophy of science pp 163-212

Ellsberg D (1963) Risk, ambiguity, and the savage axioms. The Quarterly Journal of Economics 77(2):327-336

Fishburn PC (1973) The Theory of Social Choice, vol 264. Princeton University Press Princeton

Gärdenfors P, Sahlin NE (1982) Unreliable probabilities, risk taking, and decision making. Synthese 53(3):361-386

Helzner J (2011) Uncertainty in the context of pragmatist philosophy and rational choice theory. In: Center on Capitalism and Society

Helzner J (2013) Rationalizing two-tiered choice functions through conditional choice. Synthese 190(6):929-951

Helzner J (MS) Admissibility in a logical framework, manuscript

Jeffrey RC (1983) The Logic of Decision. University of Chicago

Kadane JB, Schervish MJ, Seidenfeld T (1999) Rethinking the Foundations of Statistics. Cambridge University Press

Kahneman D, Tversky A (1979) Prospect theory: An analysis of decision under risk. Econometrica: Journal of the Econometric Society pp 263-291

Keynes JM (1921) A treatise on probability. Courier Dover Publications

Knight FH (1921) Risk, uncertainty and profit. New York: Hart, Schaffner and Marx

Kyburg HE (1968) Bets and beliefs. American Philosophical Quarterly 5(1):54-63

Levi I (1974) On indeterminate probabilities. Journal of Philosophy 71(13):391-418

Levi I (1986) Hard choices: Decision making under unresolved conflict. Cambridge University Press

Levi I (1991) The Fixation of Belief and Its Undoing: Changing Beliefs through Inquiry. Cambridge University Press 
Luce RD, Raiffa H (1957) Games and decisions: Introduction and critical survey. Courier Dover Publications

Misak C (2004) Truth and the End of Inquiry: A Peircian Account of Truth. Oxford: Clarendon Press

Moulin H (1985) Choice functions over a finite set: a summary. Social Choice and Welfare 2(2):147-160

Nehring K (1997) Rational choice and revealed preference without binariness. Social Choice and Welfare 14(3):403-425

Peirce CS (1992) The Essential Peirce, Volume 1: Selected Philosophical Writings (1867-1893), vol 1. Indiana University Press

Rott H (2001) Change, Choice and Inference: A Study of Belief Revision and Nonmonotonic Reasoning, vol 42. Oxford University Press, USA

Rubinstein A (2012) Lecture notes in microeconomic theory: the economic agent. Princeton University Press

Savage L (1972, originally published in 1954) The Foundations of Statistics. New York: John Wiley and Sons

Seidenfeld T (1988) Decision theory without independence or without ordering. Economics and Philosophy 4(2):267-290

Seidenfeld T, Kadane JB, Schervish MJ (1989) On the shared preferences of two bayesian decision makers. The Journal of Philosophy 86(5):225-244

Seidenfeld T, Schervish MJ, Kadane JB (2010) Coherent choice functions under uncertainty. Synthese 172(1):157-176

Sen A (1971) Choice functions and revealed preference. The Review of Economic Studies 38(3):307-317

Sen A (1993) Internal consistency of choice. Econometrica: Journal of the Econometric Society pp 495-521

Sen A (1997) Maximization and the act of choice. Econometrica: Journal of the Econometric Society pp 745-779

Suzumura K (1983) Rational choice, Collective decisions, and Social Welfare. Cambridge [etc.]: Cambridge University Press

Walley P (1991) Statistical reasoning with imprecise probabilities. Chapman and Hall London 